\title{
Uso de plantas como alternativa aos agrodefensivos sintéticos no cultivo de orgânicos em Goiás
}

\author{
Use of plants as an alternative to synthetic agrochemicals in the cultivation of organics in Goiás \\ Uso de plantas como alternativa a los agroquímicos sintéticos en el cultivo de orgânicos en Goiás
}

Recebido: 23/03/2021 | Revisado: 01/04/2021 | Aceito: 04/04/2021 | Publicado: 14/04/2021

Vitória Pereira Batista

ORCID: https://orcid.org/0000-0002-4506-4193 Instituto Federal de Educação, Ciência e Tecnologia Goiano, Brasil

E-mail: vitoriasd236@hotmail.com

Mônica Lau da Silva Marques

ORCID: https://orcid.org/0000-0002-2794-0815 Instituto Federal de Educação, Ciência e Tecnologia Goiano, Brasil E-mail: monica.lau@ifgoiano.edu.br

Jéssica Maria Israel de Jesus

ORCID: https://orcid.org/0000-0002-8261-9936 Universidade de São Paulo, Brasil

E-mail: jessicamariaisrael@gmail.com

Luís Sérgio Rodrigues Vale

ORCID: https://orcid.org/0000-0002-6303-9063 Instituto Federal de Educação, Ciência e Tecnologia Goiano, Brasil E-mail: luis.sergio@ifgoiano.edu.br

Eliane Vieira Rosa

ORCID: https://orcid.org/0000-0002-2144-0959 Instituto Federal de Educação, Ciência e Tecnologia Goiano, Brasil E-mail: eliane.vieira@ifgoiano.edu.br

Marta Jubielle Dias Felix

ORCID: https://orcid.org/0000-0002-2029-5083 Universidade Estadual de Goiás, Brasil E-mail: martajubielle@gmail.com

Estelita Marques

ORCID: https://orcid.org/0000-0001-7712-9687 Secretaria de Estado de Educação do Estado do Mato Grosso, Brasil

E-mail: estelita_marques31@ @otmail.com

Wanderson Moreira dos Santos

ORCID: https://orcid.org/0000-0003-1036-1945 Instituto Federal de Educação, Ciência e Tecnologia Goiano, Brasil E-mail: agro.wandsantos@gmail.com

Adriana Lau da Silva Martins

ORCID: https://orcid.org/0000-0002-0851-5522

Centro Universitário Geraldo Di Biase, Brasil E-mail: adralmartins@ hotmail.com.br

Valter dos Santos Marques

ORCID: https://orcid.org/0000-0002-9690-2361 Instituto Federal de Educação, Ciência e Tecnologia Goiano, Brasil E-mail: mltopografiavalter@gmail.com

\begin{abstract}
Resumo
Um dos principais desafios da agricultura sustentável é o controle de doenças, pragas e plantas invasoras. Diante disso, o atual estudo teve como objetivo realizar uma revisão de literatura sobre o uso de plantas como alternativa aos agrodefensivos sintéticos no cultivo orgânico em Goiás. Os defensivos naturais como o uso de plantas foram gradualmente supridos pelos sintéticos, porém recentemente, há um interesse crescente em produtos naturais para controle de doenças. O mercado de alimentos orgânicos em Goiás ainda é introvertido, porém registra desenvolvimento na produção e procura nos últimos dez anos. $\mathrm{O}$ trabalhador rural possui interesse em produzir alimentos sem utilizar agrotóxicos. A metodologia trata-se de uma revisão bibliográfica com delineamento exploratório-descritivo de caráter qualitativo. Constata-se que o uso de plantas como alternativas aos agrodefensivos são de suma importância para os agricultores, uma vez que as plantas dispõem de uma grande eficiência no confronto aos diferentes patógenos e aumenta a viabilidade do produtor. Sendo assim, considera-se que o seu uso é uma alternativa aos agrodefensivos sintéticos no desenvolvimento de produtos orgânicos na agricultura é uma estratégia ecologicamente apropriada.
\end{abstract}


Palavras-chave: Agricultura; Defensivos naturais; Sustentabilidade.

\begin{abstract}
One of the main challenges of sustainable agriculture is the control of diseases, pests and invasive plants. In view of this, the current study aimed to conduct a literature review on the use of plant as an alternative to synthetic agrodefensivos in organics cultivation in Goiás. Natural pesticides such as the use of plants have been gradually supplied by synthetics, but recently, there is a growing interest in natural products for pest control. The organic food market in Goiás is still an introvert, however it has registered growth in production and demand in the last ten years. The rural worker has an interest in producing food without using pesticides. The methodology is a bibliographic review with a qualitative exploratory-descriptive design. It is concluded that the use of plants as an alternative to agrodefensive agents, is of paramount importance for farmers, since the plants are highly effective in combating pathogenesis and increase the producer's viability. Therefore, it is considered that the use of plants as an alternative to agrodefensives in the development of organic products in agriculture is an ecologically appropriate strategy.
\end{abstract}

Keywords: Agriculture; Natural pesticides; Sustainability.

\title{
Resumen
}

Uno de los principales desafíos de la agricultura sostenible es el control de enfermedades, plagas y plantas invasoras. Ante esto, el presente estudio tuvo como objetivo realizar una revisión de la literatura sobre el uso de plantas como alternativa a los agrodefensivos sintéticos en cultivos orgánicos en Goiás. Los pesticidas naturales, como el uso de plantas, han sido suministrados gradualmente por sintéticos, pero recientemente, existe un interés creciente en productos naturales para el control de plagas. El mercado de alimentos orgánicos en Goiás sigue siendo introvertido, sin embargo, ha registrado un crecimiento en la producción y la demanda en los últimos diez años. El trabajador rural tiene interés en producir alimentos sin usar pesticidas. La metodología es una revisión bibliográfica con un diseño cualitativo exploratorio-descriptivo. Se concluye que el uso de plantas como alternativa a los agentes agrodefensivos, es de suma importancia para los agricultores, ya que las plantas son altamente efectivas en el combate de la patogenia y aumentan la viabilidad del productor. Por tanto, se considera que el uso de plantas como alternativa a los agrodefensivos en el desarrollo de productos orgánicos en la agricultura es una estrategia ecológicamente apropiada.

Palabras clave: Agricultura; Plaguicidas naturales; Sustentabilidad.

\section{Introdução}

A existência de vida na terra foi marcada pela interação de coisas vivas e o meio ambiente. Infelizmente, na busca pelo controle de pragas, o Brasil se tornou não apenas o país do futebol e do samba, entretanto do mesmo modo o maior consumidor mundial de agrotóxicos, conforme a Agência Nacional de Vigilância Sanitária (ANVISA) (Carson, 2014).

Nos últimos anos, vários modelos de produção centralizados na agroecologia têm aparecido no Brasil, contendo práticas de agricultura que incorporam as questões sociais, políticas, culturais, energéticas, ambientais e éticas, incluindo a agricultura familiar, com escala elevada de produção para o setor da agricultura orgânica. Neste sistema de produção seguemse técnicas específicas, tendo por finalidade a sustentabilidade econômica e ecológica, a maximização das benfeitorias sociais, à redução da dependência de energia não renovável, em meio a outros (Bahiense, et al., 2017).

O monitoramento de doenças nas plantações não necessita do uso excessivo de agrotóxicos. Tornando-se assim, um grande desafio para muitos agricultores. Existem alternativas para a produção de produtos de qualidade, como a utilização de plantas como alternativa aos agrodefensivos (Oliveira Júnior, et al., 2011).

Cresce a cada dia sistemas de ideias que comprovam que a procura pelo desenvolvimento sustentável integral está acoplado primeiramente ao desenvolvimento das regiões de modo sustentável (Inácio, et al., 2013). Conforme Oliveira Junior, et al., (2011), o desenvolvimento regional apenas passa a ser sustentável quando são levadas em importância os potenciais de cada região, abrangendo deste modo a economia local e as oportunidades sociais, sempre respeitando e preservando o meio ambiente.

Conforme Pádua, (2014), a precisão de ampliar sustentavelmente, a preocupação dos consumidores em consumir um alimento saudável, procedente de uma produção mais sustentável, tanto ambiental, como socialmente, passou a impulsionar a produção de alimentos orgânicos nas propriedades rurais pequenas. A produção de orgânicos passou a existir depois de determinados movimentos do final do século XIX, que repreendiam o sistema de produção da agricultura tradicional, pelos 
agravos ocasionados ao meio ambiente. No sistema orgânico o aspecto principal para eliminar as doenças e pragas é a fertilidade do solo, tratando-se de um sistema de produção retrocedido para a analogia solo-planta-ambiente (Conejero, et al., 2013).

Diante desse contexto, o presente estudo justifica-se pelo fato das enormes vantagens do consumo de alimentos orgânicos. Entretanto a prática desse sistema de cultivo ainda enfrenta grandes desafios no estado de Goiás, pois apesar das oportunidades de mercado para esses produtos, muitos agricultores carecem de melhores informações e conhecimentos técnicos para progredir com seus cultivos e comercializações.

Assim, a partir deste cenário surge o problema desta pesquisa: As plantas realmente possuem propriedades satisfatórias para combater patógenos agrícolas na produção de produtos orgânicos? Com estudo objetivou-se a realizar uma revisão de literatura sobre o uso de plantas como alternativa aos agrodefensivos sintéticos no cultivo de orgânicos em Goiás.

\section{Metodologia}

Trata-se de uma revisão bibliográfica com delineamento exploratório-descritivo de caráter qualitativo. Segundo Marconi e Lakatos (2017), abrange todo material já tornada pública em relação ao tema de estudo, desde publicações, jornais, revistas, livros, pesquisas, monografias, teses e artigos via internet. Versa-se de uma leitura atenta e sistemática que se faz várias anotações e fichamentos que servirão à fundamentação teórica do estudo.

O estudo exploratório-descritivo permite ao investigador aumentar sua experiência em torno de determinado problema e descrever com exatidão os fatos e fenômenos de determinada realidade. A pesquisa qualitativa preocupa-se com as questões particulares, ocupando-se com os significados, motivações, aspirações, atitudes, hábitos entre outros (Minayo, 2012).

Para busca de artigos científicos foram utilizadas as bases de dados: Scielo, Biblioteca Digital da Unicamp e Google Acadêmico. Os critérios de inclusão foram artigos publicados em língua portuguesa disponíveis na íntegra do ano de 2010 a 2020. E foram excluídas as publicações com mais de 10 anos, e pesquisas que não se referem ao estudo específico.

A coleta de dados foi realizada por meio da revisão da literatura somente no estudo sobre a agricultura orgânica goiana, onde todo material selecionado e estudado foi analisado. A análise de dados foi concretizada por meio dos resultados obtidos pela pesquisa bibliográfica e transcritas para o Microsoft Office Word $2013^{\circledR}$.

$\mathrm{O}$ tratamento dos dados obtidos na pesquisa é uma etapa que merece evidência, uma vez que determina a atenção dos pesquisadores referente à totalidade do processo. Assim, de posse dos resultados encontrados, com o suporte teórico escolhido foram instituídas inferências e interpretação dos referidos resultados, respondendo ao objetivo da pesquisa.

\section{Resultados e Discussão}

A sociedade tem exigido a produção de alimentos com o mínimo de degradação dos recursos naturais. Um dos principais desafios da agricultura sustentável é o controle de doenças, pragas e plantas invasoras. Assim, os agricultores acabam fazendo uso dos agrodefensivos. Segundo Conejero, et al. (2013), os agrodefensivos são substâncias químicas que surgiram com a finalidade de proteger as plantas contra as pragas ou doenças que afetam a soja, trigo, milho e outros.

Para Bahiense, et al. (2017), o problema é que esses produtos do gênero químico gera grandes impactos ambientais e à saúde dos seres vivos, podendo provocar tonturas, desmaios, dores de cabeça, câncer e podendo levar a óbito. Segundo o Ministério da Agricultura, só em 2019 foram permitidos no país 474 agrotóxicos (agrodefensivos). Esse número prova que a taxa de aprovação está cada vez mais alta e mais rápida. Representando um acréscimo de 322\% entre 2010 a 2019 (Barbosa \& Sousa, 2020).

Bahiense, et al. (2017) destaca que segundo a Agência Nacional de Vigilância Sanitária (Anvisa), o Brasil é o maior consumidor mundial de agrodefensivos. Em geral, soma em média 500 mil toneladas por ano. As características da produção 
agrícola do país justificam esse grande número. Por ser um país tropical, o aumento de pragas é influenciado diretamente por fatores climáticos, visto que a maioria das regiões não apresenta baixas temperaturas. Em suma, Conejero et al. (2013), mencionam que o uso de agrotóxicos no Brasil é regido pela Lei de Agrotóxicos $n^{\circ} 7.802$, que é regulamentada pelo Decreto $n^{\circ}$ 4.074/2002. Esta lei estabelece as limitações ao seu uso, bem como decide qual, quanto e por quem pode ser realizado.

Oliveira Júnior, et al. (2011), relata que a agricultura no Brasil, no discorrer de toda sua vivência histórica, concebe um extraordinário setor na economia brasileira, adquirindo um montante correspondendo a boa parte das exportações do País. Gomes (2016), divulga que esse aspecto tem a capacidade de contribuir para o desenvolvimento social e econômico de todo o país, uma vez que além de originar renda e emprego no meio rural, ocasiona do mesmo modo a todos os setores da economia.

Stringheta e Muniz, (2013), em suas pesquisas afirmam que agricultura orgânica pode ser determinada como um sistema holístico de gestão da produção que procura o melhoramento da biodiversidade, dos ciclos biológicos e mantimento dos solos (Zoldan \& Mior, 2017). Para Bahiense, et al. (2017), o essencial no método de produção orgânica está regressada para a sustentabilidade da agricultura, tornando mínimo os impactos ambientais, sociais e econômicos provocados pela produção agrícola.

Apesar de a produção orgânica oferecer vantagens, há a necessidade de apoio do Estado em proporcionar condições apropriadas para o desenvolvimento desse sistema. Pádua (2014) desponta determinadas dificuldades que são enfrentadas pelos agricultores de produtos orgânicos que de certo modo, dificultam o desenvolvimento da produção, como o mínimo conhecimento científico e tecnológico, e pela falta de assistência técnica de qualidade.

Conforme Sousa (2018), em 1999, no Brasil, o Ministério da Agricultura organizou a Instrução Normativa (IN) No 007/99 que determina "sistemas orgânicos de produção agropecuária" e constitui regras para a produção de produtos orgânicos, vegetais e animais. No ano de 2003, foi divulgada a Lei $\mathrm{N}^{\circ} 10.831$ que decide de modo semelhante à IN 007/99, sistema orgânico de produção agropecuária. Deste modo, Zoldan e Mior, (2017), esclarecem que muitos agricultores, não possuem conhecimento suficiente para tratar seus produtos, que muitas das vezes acabam perdendo todo seu plantio, sem saber como fazer para combater essas doenças.

Menezes (2012), diz que na agricultura, o termo patógeno é usado para se referir a qualquer tipo de microrganismo que pode causar danos às plantas. Os mais comuns são fungos, bactérias, nematóides e vírus. De acordo com a teoria, os patógenos têm a capacidade de infectar todos os tipos de plantas. No entanto, alguns patossistemas são mais agressivos do que outros. Em seguida Costa, et al., (2015), expõe que os patógenos são um dos principais motivos de perda de safra na agricultura contemporânea. $\mathrm{O}$ controle de patógenos necessita ser realizado com produtos que não prejudiquem o homem e o meio ambiente. Como nenhum pesticida é usado em produtos orgânicos, devem ser usados defensivos naturais, nos períodos com potencialidade de acontecer danos expressivos.

Souza (2018) e Costa, et al., (2015), descreve que os defensivos naturais como o uso de plantas foram gradualmente supridos pelos sintéticos, uma vez que expunham problemas como variações na eficácia, devido a diferenças na concentração do ingrediente ativo entre plantas e baixo efeito residual, que forçava a se fazer diversas aplicações em períodos curtos. Apesar disso, o interesse por produtos naturais para controle de pragas é crescente. São realizados vários estudos avaliando substâncias que apresentam baixo risco à saúde humana e ao meio ambiente, devido em parte a crescente demanda por alimentos saudáveis, sendo oriundos do sistema de produtos orgânicos (Menezes, 2012).

Corrêa e Salgado, (2011), mencionam que felizmente, são diversas plantas com atividade inseticida, muitas das quais, se possível, devem ser analisadas e inseridas nas propriedades agrícolas como modo alternativo de controle de pragas. As plantas são ricas em substâncias bioativas que incluem os carotenóides, fitoesteróis, flavonóides, fosfolipídeos, organosulfurados e polifenóis. Sendo moléculas orgânicas de baixa massa molecular que apresentam uma ampla diversidade 
química e efeitos diversos sobre organismos vivos, sendo associadas às mudanças em seu comportamento, fisiologia ou metabolismo.

Bettiol, et al. (2012), menciona que ao longo de seu desenvolvimento, algumas plantas desenvolveram mecanismos para se proteger do estresse ambiental, que vão desde a escassez de água até o ataque de pragas e infecções fúngicas e bacterianas. Uma dessas estratégias é a espuma produzida por plantas da família das sapindáceas conhecidas pela capacidade de sintetizar saponinas em suas folhas ou frutos, que nossos ancestrais usavam para lavar roupa no país. No Brasil, apesar da ausência de um levantamento minucioso sobre o uso de extratos de plantas no controle de doenças, muitos agricultores têm utilizado extratos de pimenta-do-reino, pimenta e alho. Esses extratos têm apresentando bons resultados em samambaias, eucalipto, bougainvillea entre outras plantas.

Stadnik e Talamini (2012), delineiam que também são comercializados no Brasil extratos de diversas plantas, como Bioalho ${ }^{\circledR}, \mathrm{Neemazal}^{\circledR}$ e outras. Um dos mais observados no combate às doenças das plantas é o Ecolife- $40^{\circledR}$, um extrato da biomassa cítrica contendo bioflavonóides cítricos, fitoalexinas cítrico e ácido ascórbico, que tem efeito direto na resistência e nos fitopatógenos. Recomendado contra bactérias em pimenta e morango, Botrytis cinerea em uvas, mal-de-Sigatoka em bananas e outros (O produto é registrado como fertilizante no Ministério da Agricultura, Pecuária e Abastecimento e possui selo de certificação orgânica).

A procura por produtos orgânicos foi aumentando, e assim, foi necessário aprimorar a confiança do consumidor no produto orgânico buscado. Koswoski e Cericato, (2016), mencionam que os processos de certificação são imprescindíveis para acrescentar valor ao produto, uma vez que possibilitam expandir o mercado consumidor, garantindo que o alimento seja produzido conforme a legislação vigorante para a produção orgânica. Souza, (2018), diz que a certificação é concretizada por empresas habilitadas, que classificam e padronizam as maneiras de produção segundo o conjugado de normativas e regulamentos nacionais. A certificação faz com que a produção orgânica tenha eficácia conforme determina o planejamento e documentação de modo criterioso por parte do produtor (Gomes, 2016).

Sabe-se que os consumidores estão cada vez mais obtendo interesse sobre a sustentabilidade e em alimentos mais saudáveis e estão dispostos a pagar um preço mais elevado por esses diferenciais. Entretanto, Morais, et al. (2019), expõe que a produção orgânica tem ainda um custo muito elevado pelas reivindicações de certificações e manejos de práticas culturais, embora proporcionar benfeitorias para o meio ambiente e para o trabalhador rural. O Ambiente Brasil (2016) destaca que o produto orgânico possui o preço ainda elevado pelo fato das exigências de certificações e também por ser cultivado sem a utilização de adubos químicos ou agrotóxicos. É um produto limpo, saudável, que procede de um sistema de cultivo de uma agricultura biodinâmica sendo toda a manipulação agrícola fundamentada no respeito ao meio ambiente e na preservação dos recursos naturais.

De acordo com Serviço Nacional de Aprendizagem Rural (Senar) Goiás (2017), o consumo de produtos orgânicos tem aumentado, e esse é um fator de incentivo ao produtor que segue práticas sustentáveis. Os Estados Unidos são considerados o maior mercado mundial de orgânicos, $80 \%$ das famílias consumiram produtos naturais no ano de 2016. No Brasil a percentagem é muito menor, estima-se que apenas $15 \%$ da população brasileira consomem produtos orgânicos. Em Goiás ainda não há pesquisas nesse sentido, entretanto a procura por orgânicos nas feiras livres pode-se ter uma ideia de que a demanda tem sido grande (Karan \& Zoldan, 2013).

Serviço Brasileiro de Apoio às Micro e Pequenas Empresas - Sebrae (2017), enfatiza que para que seja possível manter o quadro de desenvolvimento, cooperativas e entidades foram designadas no Estado de Goiás, para oferecer assistência ao produtor, do cultivo ao comércio de orgânicos. Elas do mesmo modo proporcionam as benfeitorias ambientais, sociais e nutricionais dos alimentos orgânicos. Um modelo é o trabalho desenvolvido pela Associação para o Desenvolvimento dos Alimentos Orgânicos de Goiás (ADAO Goiás). Instituída em 1999, a instituição embasou suas atividades em um experimento 
que obteve grande sucesso e foi desenvolvida em Fortaleza (CE), onde há uma associação com o mesmo nome e focada em estimular a prática da agricultura orgânica.

Sousa, (2018), relata que o mercado de alimentos orgânicos em Goiás ainda é introvertido, porém registra desenvolvimento na produção e procura nos últimos dez anos. Uma informação do SEBRAE em Goiás divulga o interesse do trabalhador em produzir alimentos sem utilizar agrotóxicos, entre hortaliças, frutas e raízes. Por essa razão, Oliveira e Araújo, (2018), deixam claro que a procura por plantas usadas contra patógenos agrícolas, tem aumentado cada vez mais, e em Goiás não é diferente. Algumas plantas têm propriedades inseticidas, como piretrinas, rotenona, nicotina, cevadina, veratridina, rianodina, quassinoides, azadiractina e biopesticidas voláteis. Sendo quassinoides e azadiractina, biopesticidas voláteis encontrados em óleos essenciais de plantas aromáticas (Corrêa \& Salgado, 2011).

Estudos recentes em vários países mostraram que alguns óleos essenciais de plantas não só podem espantar insetos, contudo do mesmo modo têm efeito inseticida por contato direto ou pelo trato respiratório dos insetos. Alguns ainda têm ação fungicida contra alguns patógenos importantes de plantas (Morais, et al., 2019). Um exemplo de ação inseticida é a citronela, encontrada em determinadas plantas aromáticas, como o capim limão (Cymbopogon citratus, Poaceae) e o eucalipto citriodora (Eucaliptus citriodora), sendo usadas para a produção de repelentes contra mosquitos e borrachudos (Menezes, 2012). A citronela (Cymbopogon winterianus) possui mais de 80 componentes identificados, em meio a eles o aldeído citronelal e o geraniol, que estão relacionados com o seu cheiro característico, e dispersam uma gama de insetos. Assim, partindo do conhecimento de que as plantas repelem de modo natural vários grupos de insetos existem uma grande quantidade de espécies vegetais que tem sido testada com relação a sua potencialidade como a alfavavaca (Elaeis guineenses), a hortelã (Mentha spicata) e o alecrim pimenta (Lippia sidoides) (Oliveira \& Araújo, 2018).

Rathi, et al. (2015), encontraram dez plantas com atividade inseticida, incluindo Adathoda vasica, Cynodon dactylon, Eclipta alba, Morinda pubescens, Ocimum tenuiflorum, Phyllanthus amarus, Sesbania grandifolora, Solanum surattense, Solanum trilobatum e Vinca rosea. Múltiplas misturas de solventes foram testados como extratores. Taninos e flavonóides, sendo ambos importantes membros da classe de compostos que inibem os insetos herbívoros, foram encontrados na maioria das plantas analisadas. Murugesan e Murugesh, (2013), realizaram um teste do mesmo modo com dez extratos diversos de Azadirachta indica (extrato das folhas de Neem), extrato de folhas de Pongamia glabra, extrato das folhas de Calotropis gigantea, torta de Neem, extrato das folhas de Lantana camara, óleo de Neem, Nimbecidine ${ }^{\circledR}$, extrato de folhas de Vitex negundo, extrato de folhas de Prosopis juliflora L., e extrato de alho (Allium sativum). O experimento mostrou que a população de $H$. vigintioctopunctata (besouro Hadda) diminuiu de 87,86 para 71,97\% em seguida a pulverização após o terceiro dia, e obteve os melhores resultados com óleo de Neem. No entanto, a efetividade dos extratos diminuiu nos dias seguintes à pulverização. Óleos essenciais de Neem são compostos que possuem eficácia comprovada contra um amplo espectro de pragas, e possuem baixa toxicidade para mamíferos, o que os caracteriza como seguros para a utilização em alimentos (Roychoudhury, 2010).

Seixas, (2017), enfatiza que a agricultura orgânica no Estado de Goiás é estabelecida de hortaliças, grãos, plantas medicinais e também há o desenvolvimento nos segmentos de frutas, em específico para plantios de abacate, citros, mamão, banana, caju, jabuticaba, tomate e raízes. O mercado está cada vez mais reivindicando qualidade, produtividade e preços razoáveis. Vila Verde, (2018), divulga em 2017 uma pesquisa realizada com alguns agricultores orgânicos da cidade de Goiânia em uma feira, com o intuito de compreender quais seriam as suas verdadeiras demandas e obstáculos para se sustentarem ativos e apresentarem uma postura de desenvolvimento. Os entrevistados disseram que as desvantagens em produzir orgânicos são os custos elevados com certificação e dificuldades com linha de crédito específicas. Outro obstáculo era a falta de conhecimentos suficientes sobre os defensivos naturais como o uso das plantas para combater os patógenos agrícolas (Vila Verde, 2018). 
Sousa e Goiás, (2015), destacam que se pode analisar que o procedimento de produção agrícola fundamentada na produção orgânica surge recebendo espaço em Goiás, embora esse desenvolvimento aconteça de maneira lenta e gradual. Contudo, avaliando a expansão do território e a diversidade tanto do clima quanto dos solos goianos, vê-se que o Estado evidencia condições favoráveis ao cultivo de produtos orgânicos, fazendo uso das plantas como defensivos para controlar as pragas e patógenos. Segundo Seixas (2017), ainda que o cultivo dos orgânicos seja elementar em Goiás, todavia o setor marcha para desenvolver a oferta dos produtos sem a contaminação por agrotóxicos. A produção está concentrada nos municípios de Brazabrantes, Bela Vista, Petrolina e Rio Verde, com cultivos de alface, cenoura, beterraba e tomate. Há também pequenas áreas plantadas com milho e soja orgânicos.

Sousa (2018) ressalta que no Estado de Goiás também existem muitos agricultores que possuem uma característica marcante, a preocupação referente à questão da sustentabilidade do meio rural, assim, envolvem-se no processo de desenvolvimento da agricultura orgânica no Estado, participando de fóruns e debates, na direção das associações, desenvolvimento de projetos, etc. Muitos produtores ambicionam aumentarem seus conhecimentos sobre as plantas usadas contra patógenos agrícolas. A Sociedade Nacional de Agricultura (SNA), (2018), divulga que é provável ter escala e oferecer inovação ao mercado goiano com a produção de hortaliças e frutas orgânicas, se referindo ao produtor que se encontra a frente da Fazenda da Mata, na Região Metropolitana de Goiânia para a implementação de um projeto em 440 hectares. A estrutura pioneira no Estado tem participação da Tropical Urbanismo e investimento previsto de $\mathrm{R} \$ 7$ milhões para os próximos quatro anos. Conforme Alexandria (2018), o objetivo do agricultor é se tornar um dos maiores produtores e exportadores do Brasil, e de forma sustentável, a fazenda possui $60 \%$ da sua área preservada. Portanto, pretende também mostrar que é provável produzir orgânicos por meio de defensivos naturais, com elevada qualidade e em larga escala. Atualmente, são 27 itens na linha de hortaliças e mais de 100 mil unidades produzidas por mês.

No entanto, a agricultura de produtos orgânicos goiana ainda enfrenta muitos desafios sendo um deles o da expansão dos estudos por parte das ciências agrárias, apoiando assim esse sistema de produção agrícola em condições tais que possam amparar uma população em desenvolvimento, sem elevar ainda mais a degradação e agressão do meio ambiente. Segundo Sebrae (2017), a Associação para o Desenvolvimento da Agricultura Orgânica em Goiás - ADAO/GO ainda vai vencer os desafios na agricultura, pois existem dificuldades, desde a ausência de técnicos na área, até a hora de se adaptar nos padrões da agricultura convencional. A falta de conhecimento dos agricultores, a concorrência com agroindústria/ comercialização dos produtos e a falta de conhecimentos a cerca das técnicas adequadas de produção são alguns dos desafios a serem enfrentados.

Segundo Morais, et al. (2019), a ausência de conhecimento pode afetar a evolução de muitos agricultores. Indicando assim, uma necessidade de suporte por parte das instituições representativas dos agricultores orgânicos do Estado de Goiás, referente à divulgação das informações indispensáveis, sobre a produção orgânica e agroecologia.

Contudo, vários são os desafios inerentes a ligações da cadeia produtiva, especialmente pertinentes à comercialização de produtos orgânicos, principalmente a respeito da certificação de tais produtos. Alexandria (2018) apresenta alguns dos obstáculos ao desenvolvimento do mercado de produtos orgânicos em Goiás, em meio aos quais se destacam a descontinuidade na oferta de produtos, demanda maior que à oferta, entre outros.

Entre tantos desafios, surgem às perspectivas de obter maiores orientações da produção para esse nicho de mercado. Seixas (2017), afirma que à produção orgânica se encontra em diferentes ações do governo brasileiro, que proporciona linhas de financiamento específicas para o setor e estimula projetos de transição de lavouras tradicionais para a produção orgânica. No entanto, deverá oferecer maiores conhecimentos e informações que possam abrir novos caminhos para os produtores que ainda não fazem uso de defensivos naturais a base de plantas.

De acordo com Morais, et al. (2019), a ADAO/GO desenvolve ações de acompanhamento técnico eventual nas propriedades; consultoria técnica; fiscalização da produção, formações sobre agricultura orgânica para as famílias de 
agricultores, promove e incentiva a participação dos agricultores a reuniões, seminários e "Dias de Campo". Nessas ocasiões, os consumidores são convidados, para que possam observar de perto como é realizada a produção do alimento orgânico, promovendo um trabalho de credibilidade ao procedimento de produção, que é finalizado com uso do selo da ADAO/GO nos produtos que apresentam conformidade as normas produtivas da entidade.

De acordo com a Sociedade Nacional de Agricultura (2018), apenas em Goiânia, em uma pesquisa realizada em 2017 pelo Conselho Brasileiro da Produção Orgânica e Sustentável (Organis) apontou que 22\% da população já consumiu produtos orgânicos. A principal motivação é a saúde associada ao consumo de alimentos saudáveis. A tendência, segundo o estudo, é de desenvolvimento de até $25 \%$ ao ano, o que tem feito prosperar novos negócios, inclusive em grande escala. Diante disso cresce o interesse por parte dos agricultores de produtos orgânicos em utilizar produtos naturais para o controle de pragas e doenças. Ressaltando assim a necessidade de maiores estudos e divulgação técnica a cerca da aplicabilidade e eficiência desses produtos.

\section{Considerações Finais}

Com base nas informações levantadas nesse estudo observa-se que agricultura de produtos orgânicos em Goiás, enfrenta diversos desafios como a escassez de informações sobre cultivo e a falta de assistência técnica. Verifica-se também que não existem dados concretizados a respeito do mercado de produtos orgânicos no estado.

Conforme a pesquisa bibliográfica, o uso de defensivos naturais configura-se em uma importante ferramenta de auxílio a uma produção orgânica, minimizando o impacto ambiental e a contaminação de produtores e consumidores, reduzindo os custos de produção, entre outros. Considera-se então que o uso de plantas como alternativa aos agrodefensivos sintéticos no desenvolvimento de produtos orgânicos como uma estratégia ecologicamente apropriada.

\section{Referências}

Alexandria, K. (2018). Preocupação com a saúde faz disparar produção de orgânicos. Jornal O Popular.

Ambiente Brasil. (2016). Produtos orgânicos. Ambiente Brasil.

Bahiense, D. V., Angeletti, M. P., \& Souza, J. L. (2017). Situação atual e desafios da agricultura orgânica no estado do Espírito Santo. XIX Encontro Latino Americano de Iniciação Científica, XV Encontro Latino Americano de Pós-Graduação e V Encontro de Iniciação à Docência - Universidade do Vale do Paraíba.

Barbosa, W. F., \& Sousa, E. P. (2020). Agricultura orgânica no Brasil: características e desafios. Revista Economia \& Tecnologia (RET) 8(4), 67-74.

Bettiol, W., Ghini, R., Morandi, M. A. B., Stadnik, M. J., Krauss, U., Stefanova, M., \& Prado, A. M. C. (2012). Controle biológico de doenças de plantas na América latina. In: Alves, S. B., \& Lopes, R. B. (Eds.) Controle microbiano de pragas na América Latina. Avanços e desafios. Piracicaba: FEALQ, p.303327.

Carson, R. (2014). Primavera Silenciosa. (2a ed.), Tradução Raul Polillo Edições Melhoramentos.

Conejero, M. A., Tavares, L. S., \& Neves, M. F. (2013). Produtos orgânicos: o que é, dimensões e como se habilitar. In: Neves, M. F. (Coord.) Agronegócios e desenvolvimento sustentável: uma agenda para liderança mundial na produção de alimentos e bioenergia. Atlas.

Corrêa, J. C. R., \& Salgado, H. R. N. (2011). Atividade inseticida das plantas e aplicações: revisão. Rev. Bras. Pl. Med., Botucatu, 13(4), 500-506.

Costa, E. L. N., Silva, R. F. P., \& Fiuza, L. M. (2015). Efeitos, aplicações e limitações de extratos de plantas inseticidas. Acta Biologica Leopoldensia, 26(2), $173-85$.

Gomes, J. B. P. (2016). Desafios da comercialização de produtos orgânicos oriundos da agricultura familiar no estado de Mato Grosso do Sul. Revista Brasileira de Gestão e Desenvolvimento Regional, 12(1), 132-156.

Inácio, R., Rodrigues, M., Xavier, T., Wittmann, M., \& Minussi, T. (2013). Desenvolvimento regional sustentável: abordagens para um novo paradigma. Desenvolvimento Em Questão, 11(24), 6-40. https://doi.org/10.21527/2237-6453.2013.24.6-40.

Karan, K. F., \& Zoldan, P. (2013). Comercialização e consumo de produtos agroecológicos; pesquisa dos locais de venda, pesquisa do consumidor região da grande Florianópolis: Instituto Cepa/SC, p.51. 
Research, Society and Development, v. 10, n. 4, e37910414191, 2021

(CC BY 4.0) | ISSN 2525-3409 | DOI: http://dx.doi.org/10.33448/rsd-v10i4.14191

Koswoski, K., \& Cericato, A. (2016). Desafios para a produção orgânica em propriedades rurais na agência de desenvolvimento regional (adr) de São Miguel do Oeste $-S C$.

Marconi, M. A., \& Lakatos, E. M. (2017). Fundamentos de metodologia científica. (8a ed.), Atlas.

Menezes, E. L. A. (2012). Inseticidas botânicos: seus princípios ativos, modo de ação e uso agrícola. Seropédica, Rio de Janeiro: Embrapa Agrobiologia, 58p.

Minayo, M. C. S. (2012). Pesquisa Social: teoria, método e criatividade. (18a ed.), Vozes.

Morais, F. F. S., Oliveira, M. P., Camargo, L. H. M. B., \& Caliari, R. S. (2019). Perfil dos consumidores de produtos orgânicos da feira agroecológica do mercado municipal de Goiânia-GO. Revista Verde. 7(4), 64 - 70.

Murugesan, N., \& Murugesh, T. (2013). Eficácia de alguma planta produtos contra o besouro-da-folha (besouro Hadda), Henosepilachna vigintiooctopunctata (F.) em Berinjela. Journal of Biopesticides, 1(1), 67-9.

Oliveira Junior, N., Pereira, R., \& Bresciani, L. P. (2011). Desenvolvimento Regional Sustentável no Grande ABC: análise das contribuições de uma instituição financeira brasileira. Desenvolvimento em Questão, 9(18), 49-78. https://doi.org/10.21527/2237-6453.2011.18.49-78.

Oliveira, C. J., \& Araújo, T. L. (2018). Plantas medicinais: usos e crenças de idosos portadores de hipertensão arterial. Revista Eletrônica de Farmácia, 9(1), $93-105$.

Pádua, J. B. (2014). Produção e comercialização de produtos orgânicos pela agricultura familiar em Mato Grosso do Sul. Dissertação (Mestrado em Agronegócios) Universidade Federal da Grande Dourados. Dourados-MS.

Rathi, J. M. (2015). Triagem fitoquímica qualitativa de algumas plantas inseticidas disponíveis localmente. Diário de Biopesticides, 1(1), 52-4.

Roychoudhury, R. (2016). Neem Products. In: Ecofriendly Pest Management for Food Security. Academic Press, 545-562.

Seixas, W. (2017). Orgânicos criam nicho de mercado em Goiás.

Serviço Nacional de Aprendizagem Rural - Senar Goiás. (2017). De olho no campo.

Serviço Brasileiro de Apoio às Micro e Pequenas Empresas - Sebrae. (2017). Entidades fortalecem produção de orgânicos em Goiás.

Sociedade Nacional de Agricultura - SNA. (2018). O Popular (Goiás): Preocupação com a saúde faz disparar produção de orgânicos.

Sousa, A. C. (2018). Sustentabilidade e produção orgânica: fazenda nossa senhora aparecida em Hidrolândia-GO.

Sousa, A. P., \& Goiás, B. S. (2015). Agricultura orgânica no Brasil como uma alternativa para o desenvolvimento agrícola. Revista de Economia, AnápolisGO, 11(1), 235-247.

Stadnik, M. J., \& Talamini, V. (2012). Legislação e uso de produtos naturais em países do Cone Sul. In: Stadinik, M. J., Talamini, V. (Eds.) Manejo Ecológico de Doenças de Plantas. Florianópolis, CCA/UFSC. p. 63-82.

Stringheta, P. C., \& Muniz, J. N. (2013). Alimentos orgânicos: produção, tecnologia e certificação. UFV, 452 p.

Vila Verde, E. L. N. (2018). Cesto Orgânico: Um projeto piloto na web que aproxima produtores e consumidores de orgânicos.

Zoldan, P. C., \& Mior, L. C. (2017). Produção orgânica na agricultura familiar de Santa Catarina. 94 p. (Epagri. Documentos, 239). 